\title{
A new approach toward boundedness in a two-dimensional parabolic chemotaxis system with singular sensitivity
}

\author{
Johannes Lankeit*
}

June 11, 2018

We consider the parabolic chemotaxis model

$$
\left\{\begin{array}{l}
u_{t}=\Delta u-\chi \nabla \cdot\left(\frac{u}{v} \nabla v\right) \\
v_{t}=\Delta v-v+u
\end{array}\right.
$$

in a smooth, bounded, convex two-dimensional domain and show global existence and boundedness of solutions for $\chi \in\left(0, \chi_{0}\right)$ for some $\chi_{0}>1$, thereby proving that the value $\chi=1$ is not critical in this regard.

Our main tool is consideration of the energy functional

$$
\mathcal{F}_{a, b}(u, v)=\int_{\Omega} u \ln u-a \int_{\Omega} u \ln v+b \int_{\Omega}|\nabla \sqrt{v}|^{2}
$$

for $a>0, b \geq 0$, where using nonzero values of $b$ appears to be new in this context.

Keywords: chemotaxis; singular sensitivity; global existence; boundedness

MSC: 35K55 (primary), 35A01, 35A09, 92C17, 35A07, 35B40 (secondary)

\section{Introduction}

Numerous phenomena in connection with spontaneous aggregation can be described by PDE models incorporating a cross-diffusion mechanism. A prototypical example, which lies at the core of models used for a variety of purposes and to so different aims as the description pattern formation of bacteria or slime mold in biology [11] or the prediction of burglary in criminology [14, is the following variant of the Keller-Segel system of chemotaxis:

$$
\begin{gathered}
u_{t}=\Delta u-\nabla \cdot(u S(v) \nabla v) \\
v_{t}=\Delta v-v+u \\
\left.\partial_{\nu} u\right|_{\partial \Omega}=\left.\partial_{\nu} v\right|_{\partial \Omega}=0 \\
u(\cdot, 0)=u_{0}, v(\cdot, 0)=v_{0}
\end{gathered}
$$

in a bounded domain $\Omega \subset \mathbb{R}^{n}$ with smooth boundary, with given nonnegative initial data $u_{0}, v_{0}$. We shall be concerned with the case of the singular sensitivity function $S$ given by

$$
S(v)=\frac{\chi}{v}
$$

*Institut für Mathematik, Universität Paderborn, Warburger Str. 100, 33098 Paderborn, Germany; email: johannes.lankeit@math.upb.de 
for a constant $\chi>0$, which is in compliance with the Weber-Fechner law of stimulus perception (see [12]).

One of the first questions of mathematical interest with respect to this model is that of existence of a global classical solution, as opposed to blow-up of solutions in finite time. For the vast mathematical literature on chemotaxis, a large part of which is concerned with this question, see one of the survey articles [9, 10, 7, 1] and references therein.

According to the standard reasoning in the realm of chemotaxis equations (as e.g. formulated in [1), in order to obtain global existence of classical solutions, for the two-dimensional case considered here, it is sufficient to derive $t$-independent bounds on the quantities $\int_{\Omega} u(t) \ln u(t)$ and $\int|\nabla v(t)|^{2}$.

To achieve this in the particular context of (11), it has proven useful to consider the expression

$$
\int_{\Omega} u \ln u-a \int_{\Omega} u \ln v
$$

as it has been done by Nagai, Senba, Yoshida [15] or Biler [2]. In these works, global existence of solutions has been derived for $\chi \leq 1$.

In the present article we shall answer the question whether $\chi=1$ is a critical value in this regard in the negative. This question had been left open in [20], where the above-mentioned results have been generalized to higher dimension $n$, then obtaining existence in the case $\chi<\sqrt{2 / n}$.

Let us mention some more results concerning equation (1): That the classical solutions for $\chi<\sqrt{2 / n}$ are global-in-time bounded has been shown in [4]. In [20] also weak solutions have been shown to exist for (11), as long as $\chi<\sqrt{\frac{n+2}{3 n-4}}$. In the radially symmetric setting, moreover, certain global weak "power$\lambda$-solutions" exist ([17).

Related parabolic-elliptic chemotaxis models are investigated, e.g. in [5, where the presence of terms describing logistic growth is used to ensure global existence and boundedness of classical solutions. In [6] global existence and boundedness of classical solutions to the parabolic-elliptic counterpart of (1) are obtained for even more singular sensitivities of the form $0<S(v) \leq \frac{\chi}{v^{k}}, k \geq 1$, under a smallness condition on $\chi$, which for $k=1$ and $n=2$ amounts to $\chi<1$.

Also concerning classical solutions of the fully parabolic system (1), to the best of our knowledge, the assertions for $\chi \leq 1$ are the best known so far.

Since the new possible values for $\chi$ are but slightly larger than 1 , rather than these values it is the method that can be considered the new contribution of the present article: Key to our approach toward the expansion of the interval of values for $\chi$ known to yield global solutions, namely, shall be the employment of an additional summand

$$
b \int|\nabla \sqrt{v}|^{2}
$$

in (3). Functionals containing this term have successfully been used in the context of coupled chemotaxisfluid systems (see [21]) or of chemotaxis models with consumption of the chemoattractant [18] (e.g. obtained from the aforementioned system upon neglection of the fluid).

In the end we will arrive at the following

Theorem 1.1. Let $\Omega \subset \mathbb{R}^{2}$ be a convex bounded domain with smooth boundary. Let $0 \leq u_{0} \in C^{0}(\bar{\Omega})$, $u_{0} \not \equiv 0,0<v_{0} \in \bigcup_{q>2} W^{1, q}(\Omega)$. Then there exists $\chi_{0}>1$ such that for any $\chi \in\left(0, \chi_{0}\right)$ the system (1) has a global classical solution, which is bounded.

The plan of the paper is as follows: In the next section we will discuss local existence of and an extensibility criterion for solutions to (11). Section 3 provides identities and estimates that will facilitate the usage of the additional term at the center of the proof of Theorem 1.1, to which Section 4 will be devoted.

\section{How to ensure global existence}

A general existence theorem for chemotaxis models is the following, taken from [1]: 
Theorem 2.1. Let $n \geq 1$ and $\Omega \subset \mathbb{R}^{n}$ be a bounded domain with smooth boundary and let $q>n$. For some $\omega \in(0,1)$ let $S \in C_{\text {loc }}^{1+\omega}\left(\bar{\Omega} \times[0, \infty) \times \mathbb{R}^{2}\right), f \in C^{1-}\left(\bar{\Omega} \times[0, \infty) \times \mathbb{R}^{2}\right)$ and $g \in C_{\text {loc }}^{1-}\left(\bar{\Omega} \times[0, \infty) \times \mathbb{R}^{2}\right)$, and assume that $f(x, t, 0, v) \geq 0$ for all $(x, t, v) \in \bar{\Omega} \times[0, \infty)^{2}$ and that $g(x, t, u, 0) \geq 0$ for any $(x, t, u) \in$ $\bar{\Omega} \times[0, \infty)^{2}$. Then for all nonnegative $u_{0} \in C^{0}(\bar{\Omega})$ and $v_{0} \in W^{1, q}(\Omega)$ there exist $T_{\max } \in(0, \infty]$ and $a$ uniquely determined pair of nonnegative functions

$$
\begin{aligned}
& u \in C^{0}\left(\bar{\Omega} \times\left[0, T_{\max }\right)\right) \cap C^{2,1}\left(\bar{\Omega} \times\left(0, T_{\max }\right)\right), \\
& v \in C^{0}\left(\bar{\Omega} \times\left[0, T_{\max }\right)\right) \cap C^{2,1}\left(\bar{\Omega} \times\left(0, T_{\max }\right)\right) \cap L_{l o c}^{\infty}\left(\left[0, T_{\max }\right) ; W^{1, q}(\Omega)\right),
\end{aligned}
$$

such that $(u, v)$ solves

$$
\begin{aligned}
u_{t}= & \Delta u-\nabla \cdot(u S(x, t, u, v) \nabla v)+f(x, t, u, v), \\
v_{t}= & \Delta v-v+g(x, t, u, v), \\
0= & \left.\partial_{\nu} u\right|_{\partial \Omega}=\left.\partial_{\nu} v\right|_{\partial \Omega} \\
& u(\cdot, 0)=u_{0}, v(\cdot, 0)=v_{0}
\end{aligned}
$$

classically in $\Omega \times\left(0, T_{\max }\right)$ and such that

$$
\text { if } T_{\max }<\infty, \text { then }\|u(\cdot, t)\|_{L^{\infty}(\Omega)}+\|v(\cdot, t)\|_{W^{1, q}(\Omega)} \rightarrow \infty \text { as } t \nearrow T_{\max } .
$$

Proof. A Banach-type fixed point argument provides existence of mild solutions on a short time interval whose length $T$ depends on $\left\|u_{0}\right\|_{\infty},\left\|v_{0}\right\|_{W^{1, q}}$. Standard bootstrapping arguments ensure the regularity properties listed above. It follows from the dependence of $T$ on the norms of $u_{0}$ and $v_{0}$ that the solution can be extended to $T_{\max } \in(0, \infty]$ satisfying ([6) , see [1, Lemma 4.1].

This theorem is not directly applicable to (1), because it does not cover the case of singular functions $S$. We will remove this obstruction via use of the following lemma, which is a generalization of Lemma 2.2 of [4].

Lemma 2.2. Let the conditions of Theorem 2.1 be satisfied and let $\zeta>0$.

Then there is $\eta=\eta\left(u_{0}, v_{0}, \zeta\right)>0$ such that if $v_{0}$ and the solution $(u, v)$ to (5) satisfy

$$
\inf v_{0}>0 \quad \text { and } \quad \inf _{s \in\left[0, T_{\max }\right)} \int_{\Omega} g(x, s, u(x, s), v(x, s)) d x \geq \zeta,
$$

the second component of the solution also fulfils

$$
v(x, t) \geq \eta \quad \text { for all }(x, t) \in \bar{\Omega} \times\left[0, T_{\max }\right) .
$$

Proof. Let us fix $\tau=\tau\left(u_{0}, v_{0}\right)>0$ such that

$$
\inf _{\Omega} v(\cdot, t) \geq \frac{1}{2} \inf _{\Omega} v_{0} \quad \text { for all } t \in[0, \tau]
$$

Employing the pointwise estimate

$$
\left(e^{t \Delta} w\right)(x) \geq \frac{1}{(4 \pi t)^{\frac{n}{2}}} e^{-\frac{d^{2}}{4 t}} \int_{\Omega} w \quad \text { for nonnegative } w \in C^{0}(\bar{\Omega})
$$

for the Neumann heat semigroup $e^{t \Delta}$ with $d=\operatorname{diam} \Omega$, as provided in [4, Lemma 2.2] following [8, Lemma 3.1], we can then conclude that

$$
\begin{aligned}
v(\cdot, t) & =e^{t(\Delta-1)} v_{0}+\int_{0}^{t} e^{(t-s)(\Delta-1)} g(\cdot, s, u(\cdot, s), v(\cdot, s)) d s \\
& \geq \int_{0}^{t} \frac{1}{(4 \pi(t-s))^{\frac{n}{2}}} e^{-\frac{d^{2}}{4(t-s)}-(t-s)} \int_{\Omega} g(\cdot, s, u(\cdot, s), v(\cdot, s)) d s \\
& \geq \int_{0}^{t} \frac{1}{(4 \pi r)^{\frac{n}{2}}} e^{-\left(r+\frac{d^{2}}{4 r}\right)} d r \inf _{s \in[0, t]} \int_{\Omega} g(x, s, u(x, s), v(x, s)) d x
\end{aligned}
$$




$$
\geq \zeta \int_{0}^{\tau} \frac{1}{(4 \pi r)^{\frac{n}{2}}} e^{-\left(r+\frac{d^{2}}{4 r}\right)} d r \quad \text { in } \Omega
$$

for any $t \in\left[\tau, T_{\text {max }}\right)$. With $\eta=\min \left\{\frac{\inf _{\Omega} v_{0}}{2}, \zeta \int_{0}^{\tau\left(u_{0}, v_{0}\right)} \frac{1}{(4 \pi r)^{\frac{n}{2}}} e^{-\left(r+\frac{d^{2}}{4 r}\right)} d r\right\}$ this proves the claim.

With this lemma we can weaken the assumptions on the sensitivity $S$ so as to allow for a singularity at $v=0$.

Theorem 2.3. i) Let $S \in C_{l o c}^{1+\omega}(\bar{\Omega} \times[0, \infty) \times \mathbb{R} \times(0, \infty))$ for some $\omega \in(0,1)$ and apart from the condition on $S$ let the assumptions of Theorem 2.1 be satisfied.

Additionally, assume that $f$ is nonnegative and $g(x, t, u, v) \geq c u$ for some $c>0$ and any $(x, t, u, v) \in$ $\bar{\Omega} \times[0, \infty) \times \mathbb{R}^{2}$ and that $\inf _{\Omega} v_{0}>0$ and $\int_{\Omega} u_{0}=: m>0$. Then there is $T_{\max }>0$ such that (1) has a unique solution $(u, v)$ as in (4) and such that (6) holds.

ii) Furthermore, if there are $K_{1}, K_{2}>0$ such that $f(x, t, u, v) \leq K_{1}$ and $g(x, t, u, v) \leq K_{2}(1+u)$ for all $(x, t, u, v) \in \Omega \times(0, \infty)^{3}$, and for every $\eta>0,|S|$ is bounded on $\Omega \times(0, \infty)^{2} \times(\eta, \infty)$, and if $n=2$ and there is $M>0$ such that

$$
\int_{\Omega} u(\cdot, t) \ln u(\cdot, t) \leq M, \quad \text { and } \quad \int_{\Omega}|\nabla v(\cdot, t)|^{2} \leq M \quad \text { for all } t \in\left[0, T_{\max }\right)
$$

then $(u, v)$ is global and bounded.

Proof. i) Let $\eta:=\eta\left(u_{0}, v_{0}, \mathrm{~cm}\right)$ be as in Lemma 2.2. Let $\zeta: \mathbb{R} \rightarrow[0,1]$ be a smooth, monotone decreasing function with $\zeta\left(\frac{\eta}{2}\right)=1$ and $\zeta(\eta)=0$. Define

$$
S_{\eta}(x, t, u, v):= \begin{cases}S\left(x, t, u, \frac{\eta}{2}\right), & (x, t, u, v) \in \bar{\Omega} \times[0, \infty) \times \mathbb{R} \times\left(-\infty, \frac{\eta}{2}\right), \\ \zeta(v) S\left(x, t, u, \frac{\eta}{2}\right)+(1-\zeta(v)) S(x, t, u, v), & (x, t, u, v) \in \bar{\Omega} \times[0, \infty) \times \mathbb{R} \times\left[\frac{\eta}{2}, \infty\right) .\end{cases}
$$

Then $S_{\eta} \in C_{\text {loc }}^{1+\omega}\left(\bar{\Omega} \times[0, \infty) \times \mathbb{R}^{2}\right)$ and $S$ and $S_{\eta}$ agree for $v \geq \eta$. Let us denote by (5) $\eta$ problem (5) with $S$ replaced by $S_{\eta}$. Then we can apply Theorem 2.1 to (5) $\eta$ and obtain a solution $(u, v)$ with the required properties (4) and (6). Nonnegativity of $f$ and integration of the first equation of (5) $\eta$ entail that $\int_{\Omega} u(t) \geq m$ for all $t \in\left[0, T_{\max }\right)$ and accordingly $\int_{\Omega} g(x, t, u(x, t), v(x, t)) d x \geq \mathrm{cm}>0$ for all $t \in\left[0, T_{\max }\right)$. Therefore, by Lemma 2.2, $v \geq \eta$ and hence $(u, v)$ solves (5) as well.

In order to carry over the uniqueness statement from Theorem [5, we ensure that any solution of (5) also solves (5) $\eta$ in $\Omega \times\left[0, T_{\text {max }}\right)$ : Let $v$ be a solution of (5). Let $\varepsilon \in\left(0, \frac{\eta}{2}\right)$ and define $t_{0}=\inf \left\{t: \inf _{\Omega} v(t)<\right.$ $\varepsilon\} \in(0, \infty]$. Then $(u, v)$ solves (5) $\eta$ in $\left(0, t_{0}\right)$. Assume $t_{0}<\infty$. Then by Lemma 2.2 and continuity of $v$, $v\left(x, t_{0}\right) \geq \eta>\varepsilon=\inf _{\Omega} v(\cdot, t)$ for all $x \in \Omega$, a contradiction.

ii) Since $(u, v)$ is a solution of (5) $\eta$, we can apply [1, Lemma 4.3], which turns (7) into a uniformin-time bound on $\|u(\cdot, t)\|_{L^{\infty}(\Omega)}+\|v(\cdot, t)\|_{W^{1, q}(\Omega)}$, thus asserting global existence by means of (6) and boundedness.

Remark 2.4. Throughout the remaining part of the article, we will assume that $\Omega \subset \mathbb{R}^{2}$ is a bounded, smooth domain, that $0 \leq u_{0} \in C^{0}(\bar{\Omega}), q>2$ and $v_{0} \in W^{1, q}(\Omega), \inf _{\Omega} v_{0}>0$ and $\int_{\Omega} u_{0}=: m>0$.

Then, in particular, Theorem 2.3 is applicable to (11). Furthermore, any solution $(u, v)$ of (1) satisfies

$$
\int_{\Omega} u(t)=m \quad \text { for all } t \in\left[0, T_{\max }\right) .
$$

For the purpose of using it in the next proof, let us recall the well-known Gagliardo-Nirenberg inequality:

Lemma 2.5. Let $\Omega \subset \mathbb{R}^{n}$ be a bounded smooth domain. Let $j \geq 0, k \geq 0$ be integers and $p, q, r, s>1$. There are constants $c_{1}, c_{2}>0$ such that for any function $w \in L^{q}(\Omega) \cap L^{s}(\Omega)$ with $D^{k} w \in L^{r}(\Omega)$,

$$
\left\|D^{j} w\right\|_{p} \leq c_{1}\left\|D^{k} w\right\|_{r}^{\alpha}\|w\|_{q}^{1-\alpha}+c_{2}\|w\|_{s},
$$

whenever $\frac{1}{p}=\frac{j}{n}+\left(\frac{1}{r}-\frac{k}{n}\right) \alpha+\frac{1-\alpha}{q}$ and $\frac{j}{k} \leq \alpha<1$.

Proof. Cf. [16, p. 126] 
Lemma 2.6. Let $(u, v)$ be a solution to (1), let $\tau=\min \left\{1, \frac{T_{\max }}{2}\right\}$, and assume there exists $C>0$ such that

$$
\int_{t}^{t+\tau} \int_{\Omega} \frac{|\nabla u|^{2}}{u} \leq C \quad \text { for any } t \in\left(0, T_{\max }-\tau\right)
$$

and that

$$
\int_{\Omega} u(t) \ln u(t) \leq C \quad \text { for any } t \in\left(0, T_{\max }\right)
$$

Then $T_{\max }=\infty$ and $(u, v)$ is bounded.

Proof. Let $c_{1}, c_{2}>0$ be the constants yielded by Lemma 2.5 for $j=0, k=1, q=2, r=2, \alpha=\frac{1}{2}$. Then with $m$ from (8),

$$
\begin{aligned}
\int_{t}^{t+\tau} \int_{\Omega} u^{2} & =\int_{t}^{t+\tau}\|\sqrt{u}\|_{L^{4}(\Omega)}^{4} \leq \int_{t}^{t+\tau}\left(c_{1}\|\nabla \sqrt{u}\|_{L^{2}(\Omega)}^{\alpha}\|\sqrt{u}\|_{L^{2}(\Omega)}^{1-\alpha}+c_{2}\|\sqrt{u}\|_{L^{2}(\Omega)}\right)^{4} \\
& \leq \int_{t}^{t+\tau}\left(c_{1}\left(\int_{\Omega} \frac{|\nabla u|^{2}}{4 u}\right)^{\frac{1}{4}} m^{\frac{1}{4}}+c_{2} m^{\frac{1}{2}}\right)^{4} \leq \frac{c_{1}^{4} m C}{4}+c_{2}^{4} m^{2}
\end{aligned}
$$

holds for any $t \in\left(0, T_{\max }-\tau\right)$.

Multiplying the second equation of (1) by $-\Delta v$ and integrating, from Young's inequality we obtain

$$
\int_{\Omega}|\nabla v(t)|^{2} \leq \int_{\Omega}\left|\nabla v_{0}\right|^{2}-\int_{0}^{t} \int_{\Omega}|\Delta v|^{2}-\int_{0}^{t} \int_{\Omega}|\nabla v|^{2}+\frac{1}{2} \int_{0}^{t} \int_{\Omega} u^{2}+\frac{1}{2} \int_{0}^{t} \int_{\Omega}|\Delta v|^{2} \quad \text { on }\left(0, T_{\max }\right),
$$

that is, $y(t):=\int_{\Omega}|\nabla v(t)|^{2}$ satisfies the differential inequality $y^{\prime}+y \leq f$ on $\left[0, T_{\max }\right)$, where $f=\frac{1}{2} \int_{\Omega} u^{2}$ satisfies $\int_{t}^{t+\tau}|f(s)| d s \leq C$ for all $t \in\left(0, T_{\max }-\tau\right)$ and with some constant $C>0$. Let $z$ be a solution to $z^{\prime}+z=f, z(0)=z_{0}=\int_{\Omega}\left|\nabla v_{0}\right|^{2}$ and observe that the variation-of-constants formula entails

$$
\begin{aligned}
z(t)-e^{-t} z_{0} & =\int_{0}^{t} e^{-s} f(t-s) d s \leq \sum_{k=0}^{\lfloor t / \tau\rfloor-1} \int_{k \tau}^{(k+1) \tau} e^{-s}|f(t-s)| d s+\int_{\tau\lfloor t / \tau\rfloor}^{t} e^{-s}|f(t-s)| d s \\
& \leq \sum_{k=0}^{\lfloor t / \tau\rfloor-1} e^{-k \tau} C+C \leq C\left(1+\frac{1}{1-e^{-\tau}}\right) \quad \text { for } t \in\left(0, T_{\max }\right),
\end{aligned}
$$

so that an ODE comparison yields boundedness of $y=\int_{\Omega}|\nabla v(t)|^{2}$.

Together with the second assumption, the bound on $\int_{\Omega} u \ln u$, this is sufficient to conclude global existence and boundedness of solutions by Theorem $2.3 \mathrm{ii}$ ).

\section{Some useful general estimates and identities}

Lemma 3.1. Let $\Omega$ be convex and let $w \in C^{2}(\bar{\Omega})$ satisfy $\left.\partial_{\nu} w\right|_{\partial \Omega}=0$. Then for all $x \in \partial \Omega$ also $\partial_{\nu}|\nabla w(x)|^{2} \leq 0$.

Proof. This is Lemme 2.I.1 of [13].

Lemma 3.2. For all positive $w \in C^{2}(\bar{\Omega})$ satisfying $\left.\partial_{\nu} w\right|_{\partial \Omega}=0$

$$
\int_{\Omega} w\left|D^{2} \ln w\right|^{2}=\int_{\Omega} \frac{1}{w}\left|D^{2} w\right|^{2}+\int_{\Omega} \frac{1}{w^{2}}|\nabla w|^{2} \Delta w-\int_{\Omega} \frac{|\nabla w|^{4}}{w^{3}}
$$

Proof. This proof is also contained in the proof of [21, Lemma 3.2]. The equality rests on the pointwise identity

$$
w\left|D^{2} \ln w\right|^{2}=w\left(-\frac{1}{w^{2}}|\nabla w|^{2}+\frac{1}{w} D^{2} w\right)^{2}=\frac{|\nabla w|^{4}}{w^{3}}+\frac{1}{w}\left|D^{2} w\right|^{2}-\frac{1}{w^{2}} \nabla|\nabla w|^{2} \cdot \nabla w
$$


and integration by parts in the last term giving

$$
-\int_{\Omega} \frac{1}{w^{2}} \nabla|\nabla w|^{2} \cdot \nabla w=\int_{\Omega} \frac{1}{w^{2}}|\nabla w|^{2} \Delta w-2 \int_{\Omega} \frac{|\nabla w|^{4}}{w^{3}} .
$$

Lemma 3.3. Let $w \in C^{2}(\bar{\Omega})$ be positive and satisfy $\left.\partial_{\nu} w\right|_{\partial \Omega}=0$. Then

$$
-\int_{\Omega} \frac{1}{w}|\Delta w|^{2}=-\int_{\Omega} \frac{1}{w}\left|D^{2} w\right|^{2}-\frac{3}{2} \int_{\Omega} \frac{|\nabla w|^{2} \Delta w}{w^{2}}+\frac{1}{2} \int_{\Omega} \frac{|\nabla w|^{4}}{w^{3}}+\frac{1}{2} \int_{\partial \Omega} \frac{1}{w} \partial_{\nu}|\nabla w|^{2} .
$$

Proof. This results from [21, Lemma 3.1] upon the choice of $h(w)=\frac{1}{w}$. The proof can be found in [3, Lemma 2.3].

Lemma 3.4. (i) For all positive $w \in C^{2}(\bar{\Omega})$ satisfying $\left.\partial_{\nu} w\right|_{\partial \Omega}=0$,

$$
-\int_{\Omega} \frac{1}{w}|\Delta w|^{2}=-\int_{\Omega} w\left|D^{2} \ln w\right|^{2}-\frac{1}{2} \int_{\Omega} \frac{1}{w^{2}}|\nabla w|^{2} \Delta w+\frac{1}{2} \int_{\partial \Omega} \frac{1}{w} \partial_{\nu}|\nabla w|^{2} .
$$

(ii) If furthermore $\Omega$ is convex, then

$$
-\int_{\Omega} \frac{1}{w}|\Delta w|^{2} \leq-\int_{\Omega} w\left|D^{2} \ln w\right|^{2}-\frac{1}{2} \int_{\Omega} \frac{1}{w^{2}}|\nabla w|^{2} \Delta w .
$$

Proof. This is a direct consequence of the previous three lemmata.

Lemma 3.5. There is $c_{0}>0$ such that for all positive $w \in C^{2}(\bar{\Omega})$ fulfilling $\left.\partial_{\nu} w\right|_{\partial \Omega}=0$ the following estimate holds:

$$
\int_{\Omega} w\left|D^{2} \ln w\right|^{2} \geq c_{0} \int_{\Omega} \frac{|\nabla w|^{4}}{w^{3}} .
$$

Proof. An even more general version of this lemma and its proof can be found in [21, Lemma 3.3].

Remark 3.6. As can be seen from the referenced lemma, the constant in the above statement can be chosen to be $\frac{1}{(2+\sqrt{2})^{2}}$.

\section{The energy functional. Proof of Theorem 1.1}

In this section let us investigate the energy functional defined by

$$
\mathcal{F}_{a, b}(u(t), v(t))=\int_{\Omega} u(t) \ln u(t)-a \int_{\Omega} u(t) \ln v(t)+b \int_{\Omega}|\nabla \sqrt{v(t)}|^{2}, \quad t \in\left[0, T_{\max }\right),
$$

for nonnegative parameters $a, b$.

If we want to gain useful information from this functional, the upper bounds on its derivative that we will derive, should be accompanied by bounds for $\mathcal{F}_{a, b}$ from below. In order to ensure those, let us first provide the following estimate for solutions of (1).

Lemma 4.1. Let $(u, v)$ be a solution to (11). For any $p>0$ there is $C_{p}>0$ such that

$$
\int_{\Omega} v^{p}(t) \leq C_{p} \quad \text { for any } t \in\left[0, T_{\max }\right) .
$$

Proof. Since $t \mapsto\|u(t)\|_{L^{1}(\Omega)}$ is constant by (8), for $p \geq 1$ this is a consequence of Duhamel's formula for the solution of the second equation of (1) and estimates for the Neumann heat semigroup, which can e.g. be found in [19, Lemma 1.3]: They provide $C>0$ such that for all $t \in\left(0, T_{\max }\right)$,

$$
\begin{aligned}
\|v(t)\|_{L^{p}(\Omega)} & \leq\left\|e^{t(\Delta-1)} v_{0}\right\|_{L^{p}(\Omega)}+\int_{0}^{t}\left\|e^{(t-s)(\Delta-1)}(u(s)-m)\right\|_{L^{p}(\Omega)}+\left\|e^{-(t-s)} m\right\|_{L^{p}(\Omega)} d s \\
& \leq\left\|v_{0}\right\|_{L^{p}(\Omega)}+\int_{0}^{t}\left(C\left(1+(t-s)^{-\frac{n}{2}\left(1-\frac{1}{p}\right)}\right) e^{-(t-s)}\|u(s)-m\|_{L^{1}(\Omega)}+e^{-(t-s)} m|\Omega|^{\frac{1}{p}}\right) d s .
\end{aligned}
$$

The case $p \in(0,1)$ then follows from $v^{p} \leq 1+v$. 
The following lemma gives bounds from below as well as means to turn boundedness of $\mathcal{F}_{a, b}(u, v)$ into boundedness of $\int_{\Omega} u \ln u$.

Lemma 4.2. Let $a, b \geq 0$. For any solution $(u, v)$ of (1), there is $\gamma \in \mathbb{R}$ such that

$$
\mathcal{F}_{a, b}(u, v) \geq \frac{1}{2} \int_{\Omega} u \ln u-\gamma \quad \text { on }\left(0, T_{\max }\right)
$$

Proof. Denoting $m=\int_{\Omega} u(t)$ as in (8), we have

$$
\mathcal{F}_{a, b}(u, v) \geq \frac{1}{2} \int_{\Omega} u \ln u+\int_{\Omega} u \ln \frac{u^{\frac{1}{2}}}{v^{a}}=\frac{1}{2} \int_{\Omega} u \ln u+m \int_{\Omega}\left(-\ln \frac{v^{a}}{u^{\frac{1}{2}}}\right) \frac{u}{m},
$$

similar as in the proof of [2, Thm. 3]. Hence, following an idea from the proof of [15, Lemma 3.3] in applying Jensen's inequality with the probability measure $\frac{u}{m} d \lambda$ and the convex function $-\ln$, we obtain

$$
\begin{aligned}
\mathcal{F}_{a, b}(u, v) & \geq \frac{1}{2} \int_{\Omega} u \ln u-m \ln \int_{\Omega} \frac{v^{a} u^{\frac{1}{2}}}{m} \\
& \geq \frac{1}{2} \int_{\Omega} u \ln u-m \ln \left(\frac{1}{m}\left(\int_{\Omega} v^{2 a} \int_{\Omega} u\right)^{\frac{1}{2}}\right) \\
& \geq \frac{1}{2} \int_{\Omega} u \ln u+\frac{m}{2} \ln m-\frac{m}{2} \ln C_{2 a}
\end{aligned}
$$

after applying Hölder's inequality and with $C_{2 a}$ as in 4.1

Lemma 4.3. Let $a, b \geq 0$. For any solution $(u, v)$ of (1),

i) $\mathcal{F}_{a, b}(u, v)$ is bounded below.

ii) If $\sup _{t \in\left[0, T_{\max }\right)} \mathcal{F}_{a, b}(u(t), v(t))<\infty$ then $\sup _{t \in\left[0, T_{\max }\right)} \int_{\Omega} u(t) \ln u(t)<\infty$.

Proof. Both statements are immediate consequences of Lemma 4.2.

Lemma 4.1 as well enables us to control the first two summands of $\mathcal{F}_{a, b}(u, v)$ from above by $\int_{\Omega} \frac{u^{2}}{v}$.

Lemma 4.4. Let $(u, v)$ be a solution to (11) and let $a>0$. Then for any $\delta>0$ there is $c_{\delta}>0$ such that

$$
\int_{\Omega} u \ln u-a \int_{\Omega} u \ln v \leq \delta \int_{\Omega} \frac{u^{2}}{v}+c_{\delta} \quad \text { on }\left[0, T_{\max }\right) .
$$

Proof. Given $a>0$ let $\varepsilon \in(0,1)$ be so small that $\frac{1+\varepsilon-2 a \varepsilon}{1-\varepsilon}>0$. There is $C_{\varepsilon}>0$ such that for any $x>0$ we have $\ln x \leq C_{\varepsilon} x^{\varepsilon}$. Therefore for any $\delta>0$ Young's inequality and Lemma 4.1 provide $C_{\delta}>0$ and $c_{\delta}>0$ satisfying

$$
\begin{aligned}
\int_{\Omega} u \ln u-a \int_{\Omega} u \ln v & =\int_{\Omega} u \ln \frac{u}{v^{a}} \leq C_{\varepsilon} \int_{\Omega} \frac{u^{1+\varepsilon}}{v^{a \varepsilon}} \leq \delta \int_{\Omega}\left(u^{1+\varepsilon} v^{-\frac{1+\varepsilon}{2}}\right)^{\frac{2}{1+\varepsilon}}+C_{\delta} \int_{\Omega}\left(v^{\frac{1+\varepsilon-2 a \varepsilon}{2}}\right)^{\frac{2}{1-\varepsilon}} \\
& \leq \delta \int_{\Omega} \frac{u^{2}}{v}+c_{\delta}
\end{aligned}
$$

With these preparations, we turn to the time derivative of $\mathcal{F}_{a, b}(u, v)$, beginning with the already investigated first part:

Lemma 4.5. For any $a \geq 0$ and any solution $(u, v)$ of (1),

$$
\frac{\mathrm{d}}{\mathrm{d} t} \mathcal{F}_{a, 0}(u, v)(t)=-\int_{\Omega} \frac{|\nabla u|^{2}}{u}+(\chi+2 a) \int_{\Omega} \frac{\nabla u \cdot \nabla v}{v}-a(\chi+1) \int_{\Omega} \frac{u|\nabla v|^{2}}{v^{2}}+a \int_{\Omega} u-a \int_{\Omega} \frac{u^{2}}{v}
$$

holds on $\left(0, T_{\max }\right)$. 
Proof. Using the first equation of (11) in $\frac{\mathrm{d}}{\mathrm{d} t}\left(\int_{\Omega} u \ln u-a \int_{\Omega} u \ln v\right)$ and integrating by parts we obtain:

$$
\begin{aligned}
\frac{\mathrm{d}}{\mathrm{d} t}\left(\int_{\Omega} u \ln u-a \int_{\Omega} u \ln v\right)= & \int_{\Omega} u_{t} \ln u+\int_{\Omega} u_{t}-a \int_{\Omega} u_{t} \ln v-a \int_{\Omega} \frac{u}{v} v_{t} \\
= & -\int_{\Omega} \frac{\nabla u}{u}\left(\nabla u-\chi \frac{u}{v} \nabla v\right)+a \int_{\Omega} \frac{\nabla v}{v}\left(\nabla u-\chi \frac{u}{v} \nabla v\right)-a \int_{\Omega} \frac{u}{v}(\Delta v-v+u) \\
= & -\int_{\Omega} \frac{|\nabla u|^{2}}{u}+\chi \int_{\Omega} \frac{\nabla u \cdot \nabla v}{v}+a \int_{\Omega} \frac{\nabla u \cdot \nabla v}{v}-a \chi \int_{\Omega} \frac{u|\nabla v|^{2}}{v^{2}} \\
& +a \int_{\Omega} \frac{\nabla u \cdot \nabla v}{v}-a \int_{\Omega} \frac{u|\nabla v|^{2}}{v^{2}}+a \int_{\Omega} u-a \int_{\Omega} \frac{u^{2}}{v}
\end{aligned}
$$

Since we do not know the sign of $\int_{\Omega} \frac{\nabla u \cdot \nabla v}{v}$ and, in this situation, cannot control $\int_{\Omega} \frac{u|\nabla v|^{2}}{v^{2}}$, we are left with Young's inequality, hoping that the resulting coefficient $\frac{(\chi+2 a)^{2}}{4}-a(\chi+1)$ of $\int_{\Omega} \frac{u|\nabla v|^{2}}{v^{2}}$ turns out to be negative. This can be achieved if $\chi<1$.

However, it becomes possible to cope with larger parameters if $\int_{\Omega} \frac{u|\nabla v|^{2}}{v^{2}}$ can be controlled, e.g. by having control over $\int_{\Omega} \frac{|\nabla v|^{4}}{v^{3}}$ and $\int_{\Omega} \frac{u^{2}}{v}$. The second term already being in place, fortunately, the first is one of the terms arising from the following:

Lemma 4.6. Let $\Omega$ be convex. For any solution $(u, v)$ of (1),

$$
4 \frac{\mathrm{d}}{\mathrm{d} t}\left(\int_{\Omega}|\nabla \sqrt{v}|^{2}\right) \leq-2 c_{0} \int_{\Omega} \frac{|\nabla v|^{4}}{v^{3}}-\int_{\Omega} \frac{|\nabla v|^{2}}{v}+2 \int_{\Omega} \frac{\nabla u \cdot \nabla v}{v}-\int_{\Omega} \frac{|\nabla v|^{2} u}{v^{2}}
$$

holds on $\left(0, T_{\max }\right)$, where $c_{0}$ is the constant provided by Lemma 3.5.

Proof. From the second equation of (1), we obtain

$$
\begin{aligned}
4 \frac{\mathrm{d}}{\mathrm{d} t}\left(\int_{\Omega}|\nabla \sqrt{v}|^{2}\right) & =\frac{\mathrm{d}}{\mathrm{d} t} \int_{\Omega} \frac{|\nabla v|^{2}}{v}=\int_{\Omega} \frac{2 \nabla v \cdot \nabla v_{t}}{v}-\int_{\Omega} \frac{|\nabla v|^{2} v_{t}}{v^{2}} \\
& =\int_{\Omega} \frac{2 \nabla v \cdot \nabla \Delta v}{v}-\int_{\Omega} \frac{2|\nabla v|^{2}}{v}+\int_{\Omega} \frac{2 \nabla v \cdot \nabla u}{v}-\int_{\Omega} \frac{|\nabla v|^{2} \Delta v}{v^{2}}+\int_{\Omega} \frac{|\nabla v|^{2} v}{v^{2}}-\int_{\Omega} \frac{|\nabla v|^{2} u}{v^{2}} .
\end{aligned}
$$

Integration by parts in the first integral and merging the second and second to last summand lead us to

$$
4 \frac{\mathrm{d}}{\mathrm{d} t}\left(\int_{\Omega}|\nabla \sqrt{v}|^{2}\right)=-2 \int_{\Omega} \frac{|\Delta v|^{2}}{v}+\int_{\Omega} \frac{|\nabla v|^{2} \Delta v}{v^{2}}-\int_{\Omega} \frac{|\nabla v|^{2}}{v}+2 \int_{\Omega} \frac{\nabla u \cdot \nabla v}{v}-\int_{\Omega} \frac{|\nabla v|^{2} u}{v^{2}} .
$$

By Lemma 3.4 and due to the convexity of $\Omega$ we can transform the first summand according to

$$
-2 \int_{\Omega} \frac{|\Delta v|^{2}}{v} \leq-2 \int_{\Omega} v\left|D^{2} \ln v\right|^{2}-\int_{\Omega} \frac{1}{v^{2}}|\nabla v|^{2} \Delta v
$$

making the second term in the right hand side of (11) vanish:

$$
4 \frac{\mathrm{d}}{\mathrm{d} t}\left(\int_{\Omega}|\nabla \sqrt{v}|^{2}\right) \leq-2 \int_{\Omega} v\left|D^{2} \ln v\right|^{2}+2 \int_{\Omega} \frac{\nabla u \cdot \nabla v}{v}-\int_{\Omega} \frac{|\nabla v|^{2} u}{v^{2}} .
$$

We are left with a term we can estimate with the help of Lemma 3.5.

$$
-2 \int_{\Omega} v\left|D^{2} \ln v\right|^{2} \leq-2 c_{0} \int_{\Omega} \frac{|\nabla v|^{4}}{v^{3}}
$$

thereby gaining the term which will make the crucial difference in the estimates to come and arriving at

$$
4 \frac{\mathrm{d}}{\mathrm{d} t}\left(\int_{\Omega}|\nabla \sqrt{v}|^{2}\right) \leq-2 c_{0} \int_{\Omega} \frac{|\nabla v|^{4}}{v^{3}}-\int_{\Omega} \frac{|\nabla v|^{2}}{v}+2 \int_{\Omega} \frac{\nabla u \cdot \nabla v}{v}-\int_{\Omega} \frac{|\nabla v|^{2} u}{v^{2}} .
$$


If we combine the previous two lemmata, we are led to:

Lemma 4.7. Let $\Omega \subset \mathbb{R}^{2}$ be a convex, bounded, smooth domain and let $a, b \geq 0, \delta \in(0,1)$. Then for any solution $(u, v)$ of (1),

$$
\begin{aligned}
\frac{\mathrm{d}}{\mathrm{d} t} \mathcal{F}_{a, b}(u, v)(t) \leq & \left.\frac{1}{4 a(1-\delta)}\left(\frac{\left(\chi+2 a+\frac{b}{2}\right)^{2}}{4(1-\delta)}-a \chi-a-\frac{b}{4}\right)_{+}^{2}-\frac{b c_{0}}{2}\right) \int_{\Omega} \frac{|\nabla v|^{4}}{v^{3}} \\
& -\delta \int_{\Omega} \frac{|\nabla u|^{2}}{u}-\delta \int_{\Omega} \frac{u^{2}}{v}+a \int_{\Omega} u-\frac{b}{4} \int_{\Omega} \frac{|\nabla v|^{2}}{v} \quad \text { on }\left(0, T_{\max }\right) .
\end{aligned}
$$

Proof. An estimate for $\frac{\mathrm{d}}{\mathrm{d} t} \mathcal{F}_{a, b}(u(t), v(t))$ is given by the sum of the terms from Lemma 4.5 and Lemma 4.6 .

$$
\begin{aligned}
\frac{\mathrm{d}}{\mathrm{d} t} \mathcal{F}_{a, b}(u, v)(t) \leq & -\int_{\Omega} \frac{|\nabla u|^{2}}{u}+\left(\chi+2 a+\frac{b}{2}\right) \int_{\Omega} \frac{\nabla u \cdot \nabla v}{v}-\left(a \chi+a+\frac{b}{4}\right) \int_{\Omega} \frac{u|\nabla v|^{2}}{v^{2}} \\
& +a \int_{\Omega} u-a \int_{\Omega} \frac{u^{2}}{v}-\frac{b}{2} c_{0} \int_{\Omega} \frac{|\nabla v|^{4}}{v^{3}}-\frac{b}{4} \int_{\Omega} \frac{|\nabla v|^{2}}{v} .
\end{aligned}
$$

In order to finally still have some control over $\int \frac{|\nabla u|^{2}}{u}$, as required for Lemma 2.6, we retain a small portion of this term when applying Young's inequality:

$$
-\int_{\Omega} \frac{|\nabla u|^{2}}{u}+\left(\chi+2 a+\frac{b}{2}\right) \int_{\Omega} \frac{\nabla u \cdot \nabla v}{v} \leq(-1+(1-\delta)) \int_{\Omega} \frac{|\nabla u|^{2}}{u}+\frac{\left(\chi+2 a+\frac{b}{2}\right)^{2}}{4(1-\delta)} \int_{\Omega} \frac{u|\nabla v|^{2}}{v^{2}}
$$

so that

$$
\begin{aligned}
\frac{\mathrm{d}}{\mathrm{d} t} \mathcal{F}_{a, b}(u, v)(t) \leq & -\delta \int_{\Omega} \frac{|\nabla u|^{2}}{u}+\left(\frac{\left(\chi+2 a+\frac{b}{2}\right)^{2}}{4(1-\delta)}-a \chi-a-\frac{b}{4}\right) \int_{\Omega} \frac{u|\nabla v|^{2}}{v^{2}} \\
& +a \int_{\Omega} u-a \int_{\Omega} \frac{u^{2}}{v}-\frac{b}{2} c_{0} \int_{\Omega} \frac{|\nabla v|^{4}}{v^{3}}-\frac{b}{4} \int_{\Omega} \frac{|\nabla v|^{2}}{v}
\end{aligned}
$$

By virtue of the presence of $-\int_{\Omega} \frac{|\nabla v|^{4}}{v^{3}}$, which originates from the additional summand of the energy functional and the preparations of Section 3 , we can continue estimating $\int_{\Omega} \frac{u|\nabla v|^{2}}{v^{2}}$ by $\int_{\Omega} \frac{u^{2}}{v}$ and $\int_{\Omega} \frac{|\nabla v|^{4}}{v^{3}}$ and still hope for negative coefficients in front of the integrals, in contrast to the situation of Lemma 4.5 In doing so we keep some part of $\int_{\Omega} \frac{u^{2}}{v}$ for the sake of a later application of Lemma 4.4 and arrive at

$$
\begin{aligned}
\frac{\mathrm{d}}{\mathrm{d} t} \mathcal{F}_{a, b}(u, v)(t) \leq & -\delta \int_{\Omega} \frac{|\nabla u|^{2}}{u}+a(1-\delta) \int_{\Omega} \frac{u^{2}}{v}+\frac{1}{4 a(1-\delta)}\left(\frac{\left(\chi+2 a+\frac{b}{2}\right)^{2}}{4(1-\delta)}-a \chi-a-\frac{b}{4}\right)_{+}^{2} \int_{\Omega} \frac{|\nabla v|^{4}}{v^{3}} \\
& +a \int_{\Omega} u-a \int_{\Omega} \frac{u^{2}}{v}-\frac{b}{2} c_{0} \int_{\Omega} \frac{|\nabla v|^{4}}{v^{3}}-\frac{b}{4} \int_{\Omega} \frac{|\nabla v|^{2}}{v}
\end{aligned}
$$

which amounts to (13).

Lemma 4.8. Let $a>0, b \geq 0, \chi>0$ be such that

$$
\varphi(a, b ; \chi):=\left(\frac{1}{4 a}\left(\frac{\left(\chi+2 a+\frac{b}{2}\right)^{2}}{4}-a \chi-a-\frac{b}{4}\right)_{+}^{2}-\frac{b c_{0}}{2}\right)<0
$$

and let $(u, v)$ be a solution of (11). Then there are $\kappa, \delta>0$ and $c>0$ such that for any $t \in\left(0, T_{\max }\right)$,

$$
\frac{\mathrm{d}}{\mathrm{d} t} \mathcal{F}_{a, b}(u, v)(t)+\kappa \mathcal{F}_{a, b}(u, v)(t)+\delta \int_{\Omega} \frac{|\nabla u(t)|^{2}}{u(t)} \leq c .
$$


Proof. By continuity of

$$
\delta \mapsto \varphi_{\delta}(a, b ; \chi):=\left(\frac{1}{4 a(1-\delta)}\left(\frac{\left(\chi+2 a+\frac{b}{2}\right)^{2}}{4(1-\delta)}-a \chi-a-\frac{b}{4}\right)_{+}^{2}-\frac{b c_{0}}{2}\right)
$$

in $\delta=0$, for fixed $a, b, \chi$, negativity of $\varphi(a, b ; \chi)$ entails the existence of $\delta>0$ so that $\varphi_{\delta}(a, b ; \chi)$ is negative as well. Therefore, by Lemma 4.7.

$$
\frac{\mathrm{d}}{\mathrm{d} t} \mathcal{F}_{a, b}(u, v)+\delta \int_{\Omega} \frac{|\nabla u|^{2}}{u}+\delta \int_{\Omega} \frac{u^{2}}{v}+b \int_{\Omega}|\nabla \sqrt{v}|^{2} \leq a \int_{\Omega} u \quad \text { on }\left(0, T_{\max }\right) .
$$

Since $\int_{\Omega} u$ is constant in time by (8), Lemma 4.4 implies the assertion.

Lemma 4.9. If

$$
\chi_{0} \in\{\chi>0 ; \text { there are } a>0 \text { and } b \geq 0 \text { such that } \varphi(a, b ; \chi)<0\}=: M,
$$

then

$$
\left(0, \chi_{0}\right) \subset M
$$

Proof. Since, for any fixed $a>0, b \geq 0$,

$$
\chi \mapsto \varphi(a, b ; \chi)=\frac{1}{64 a}\left(\left(\chi^{2}+4 a^{2}+\frac{b^{2}}{4}+b \chi+2 a b-4 a-b\right)_{+}^{2}-32 a b c_{0}\right)
$$

is monotone, for any $a>0, b \geq 0$

$$
\varphi\left(a, b ; \chi_{0}\right)<0 \text { implies } \varphi(a, b ; \chi)<0 \text { for any } 0<\chi<\chi_{0} .
$$

Lemma 4.10. There is $\chi_{0}>1$ such that $\varphi\left(a, b ; \chi_{0}\right)<0$ for some $a>0, b>0$.

Proof. Since $\varphi\left(\frac{1}{2}, 0,1\right)=0$ and

$$
\left.\frac{d}{d b} \varphi\left(\frac{1}{2}, b, 1\right)\right|_{b=0}=\left.\frac{d}{d b}\left(\frac{1}{32}\left(\frac{b^{2}}{4}+b\right)^{2}-\frac{1}{2} c_{0} b\right)\right|_{b=0}=\left.\left[\frac{1}{16}\left(\frac{b^{2}}{4}+b\right)\left(\frac{b}{2}+1\right)-\frac{1}{2} c_{0}\right]\right|_{b=0}=-\frac{c_{0}}{2}<0
$$

there is $b>0$ such that $\varphi\left(\frac{1}{2}, b, 1\right)<0$ and by continuity of $\varphi$ with respect to $\chi$, the assertion follows.

Proof of Theorem 1.1. By Lemma 4.10, there are $a, b>0, \chi_{0}>1$ such that $\varphi\left(a, b, \chi_{0}\right)<0$ and hence, by Lemma 4.9, also $\varphi(a, b, \chi)<0$ for $\chi \in\left(0, \chi_{0}\right)$. An application of Lemma 4.8 thus reveals that for all $t>0$

$$
\frac{\mathrm{d}}{\mathrm{d} t} \mathcal{F}_{a, b}(u, v)(t)+\kappa \mathcal{F}_{a, b}(u, v)(t)+\delta \int_{\Omega} \frac{|\nabla u|^{2}}{u} \leq c
$$

for some $\kappa, \delta, c>0$. Together with the boundedness of $\mathcal{F}_{a, b}(u, v)$ from below by Lemma $4.3 \mathrm{i}$ ) this ensures that $\mathcal{F}_{a, b}(u, v)$ is bounded so that an integration of (15) also shows the boundedness of $\int_{t}^{t+1} \int_{\Omega} \frac{|\nabla u|^{2}}{u}$. Since $\mathcal{F}_{a, b}(u, v)$ is bounded, by Lemma 4.3 ii) the same holds true for $\int_{\Omega} u \ln u$ and so the conditions of Lemma 2.6 are met and Theorem 1.1 follows.

Remark 4.11. Assuming $c_{0}=\frac{1}{(2+\sqrt{2})^{2}}$, as permitted by Remark 3.6,

$$
-1.1 \cdot 10^{-5} \approx \varphi(0.49,0.001 ; 1.015)<0,
$$

i.e. $\chi_{0}>1.015$. 


\section{References}

[1] N. Bellomo, A. Bellouquid, Y. Tao, and M. Winkler. Toward a mathematical theory of Keller-Segel models of pattern formation in biological tissues. preprint.

[2] P. Biler. Global solutions to some parabolic-elliptic systems of chemotaxis. Adv. Math. Sci. Appl., 9(1):347359, 1999.

[3] R. Dal Passo, H. Garcke, and G. Grün. On a fourth-order degenerate parabolic equation: Global entropy estimates, existence, and qualitative behavior of solutions. SIAM J. Math. Anal., 29(2):321-342, 1998.

[4] K. Fujie. Boundedness in a fully parabolic chemotaxis system with singular sensitivity. J. Math. Anal. Appl., 424(1):675 - 684, 2015.

[5] K. Fujie, M. Winkler, and T. Yokota. Blow-up prevention by logistic sources in a parabolic-elliptic KellerSegel system with singular sensitivity. Nonlinear Anal. Theory, Meth. Appl., 109(0):56 - 71, 2014.

[6] K. Fujie, M. Winkler, and T. Yokota. Boundedness of solutions to parabolic-elliptic Keller-Segel systems with signal-dependent sensitivity. Math. Methods Appl. Sci., pages n/a-n/a, 2014.

[7] T. Hillen and K. J. Painter. A user's guide to PDE models for chemotaxis. J. Math. Biol., 58(1-2):183-217, 2009.

[8] T. Hillen, K. J. Painter, and M. Winkler. Convergence of a cancer invasion model to a logistic chemotaxis model. Math. Models Methods Appl. Sci., 23(1):165-198, 2013.

[9] D. Horstmann. From 1970 until present: the Keller-Segel model in chemotaxis and its consequences. I. Jahresber. Deutsch. Math.-Verein., 105(3):103-165, 2003.

[10] D. Horstmann. From 1970 until present: the Keller-Segel model in chemotaxis and its consequences. II. Jahresber. Deutsch. Math.-Verein., 106(2):51-69, 2004.

[11] E. F. Keller and L. A. Segel. Initiation of slime mold aggregation viewed as an instability. J. Theor. Biol., 26(3):399-415, 1970.

[12] E. F. Keller and L. A. Segel. Traveling bands of chemotactic bacteria: A theoretical analysis. J. Theor. Biol., 30(2):235 - 248, 1971.

[13] P. Lions. Résolution de problèmes elliptiques quasilinéaires. Arch. Rational Mech. Anal., 74(4):335-353, 1980.

[14] R. Manásevich, Q. H. Phan, and Ph. Souplet. Global existence of solutions for a chemotaxis-type system arising in crime modelling. European J. Appl. Math., 24(2):273-296, 2013.

[15] T. Nagai, T. Senba, and K. Yoshida. Global existence of solutions to the parabolic systems of chemotaxis. Sürikaisekikenkyūsho Kökyūroku, (1009):22-28, 1997.

[16] L. Nirenberg. On elliptic partial differential equations. Ann. Sc. Norm. Super. Pisa, Sci. Fis. Mat., III. Ser., 13:115-162, 1959.

[17] C. Stinner and M. Winkler. Global weak solutions in a chemotaxis system with large singular sensitivity. Nonlinear Anal. Real World Appl., 12(6):3727 - 3740, 2011.

[18] Y. Tao and M. Winkler. Eventual smoothness and stabilization of large-data solutions in a three-dimensional chemotaxis system with consumption of chemoattractant. J. Differential Equations, 252(3):2520-2543, 2012.

[19] M. Winkler. Aggregation vs. global diffusive behavior in the higher-dimensional Keller-Segel model. $J$. Differential Equations, 248(12):2889-2905, 2010.

[20] M. Winkler. Global solutions in a fully parabolic chemotaxis system with singular sensitivity. Math. Methods Appl. Sci., 34(2):176-190, 2011.

[21] M. Winkler. Global large-data solutions in a chemotaxis-(Navier-)Stokes system modeling cellular swimming in fluid drops. Comm. Partial Differential Equations, 37(2):319-351, 2012. 